\title{
Laplace Discrete Adomian Decomposition Method for Solving Nonlinear Integro Differential Equations
}

\author{
H. O. Bakodah', M. Al-Mazmumy', S. O. Almuhalbedi' ${ }^{2}$, Lazim Abdullah ${ }^{3}$ \\ ${ }^{1}$ Department of Mathematics, Faculty of Science, University of Jeddah, Jeddah, KSA \\ ${ }^{2}$ Department of Mathematics; Faculty of Science; King Abdulaziz University, Jeddah, KSA \\ ${ }^{3}$ School of Informatics and Applied Mathematics, Universiti Malaysia Terengganu, Kuala Terengganu, Malaysia \\ Email: hobakodah@uj.edu.sa,mhalmazmumy@uj.edu.sa,sss-2011-s@hotmail.com, lazim_m@umt.edu.my
}

How to cite this paper: Bakodah, H.O., Al-Mazmumy, M., Almuhalbedi, S.O. and Abdullah, L. (2019) Laplace Discrete Adomian Decomposition Method for Solving Nonlinear Integro Differential Equations. Journal of Applied Mathematics and Physics, 7, 1388-1407.

https://doi.org/10.4236/jamp.2019.76093

Received: June 29, 2018

Accepted: June 27, 2019

Published: June 30, 2019

Copyright $\odot 2019$ by author(s) and Scientific Research Publishing Inc. This work is licensed under the Creative Commons Attribution International License (CC BY 4.0).

http://creativecommons.org/licenses/by/4.0/

\begin{abstract}
This paper proposes the Laplace Discrete Adomian Decomposition Method and its application for solving nonlinear integro-differential equations. This method is based upon the Laplace Adomian decomposition method coupled with some quadrature rules of numerical integration. Four numerical examples of integro-differential equations in both Volterra and Fredholm integrals are used to be solved by the proposed method. The performance of the proposed method is verified through absolute error measures between the approximated solutions and exact solutions. The series of experimental numerical results show that our proposed method performs in high accuracy and efficiency. The study clearly highlights that the proposed method could be used to overcome the analytical approaches in solving nonlinear integro-differential equations.
\end{abstract}

\section{Keywords}

Integro-Differential Equation, Volterra Integro-Differential Equation, Fredholm Integro-Differential Equation, Laplace Adomian Decomposition Method, Quadrature Rules

\section{Introduction}

One of the issues in finding the solutions of integro-differential equations is the choices of approaches used that are either analytical or numerical. Previous literature suggested that analytical solutions of integro-differential equations are not usually obtainable especially when the equations are entangled with nonlinear terms. Therefore, it is required to find an efficient approximate solution 
numerically. Much attention has been devoted for the search of better and more efficient methods in recent years with the introduction of several numerical methods such as the Galerkin methods [1], Runge-Kutta methods [2], Chebyshev collocation method [3], Taylor collection method [4], rationalized Haar functions method [5], Galerkin methods with hybrid functions [6], and Adomian Decomposition Method (ADM) [7]-[12]. In addition to these numerical methods, Khuri [13] [14] used Laplace transform numerical scheme, based on the $\mathrm{ADM}$ to solve nonlinear differential equations and Bratu's problem, respectively. This method is popularly known as Laplace Adomian Decomposition Method (LADM) where the ADM and Laplace transforms are combined.

The LADM is known for its rapid convergence in solution and also uses only little iteration as successfully applied in Kiymaz [15]. Furthermore, several modifications of the ADM and LADM methods can be seen in [16]-[22] with wide applications ranging from differential equations, partial differential equations, integral equations and integro-differential equations among others. It seems that the LADM method is always open for further modifications especially on discretizing the Adomian decomposition. In this paper, we aim at extending the Laplace Adomian decomposition method for finding the solution of nonlinear integro-differential equations by firstly discretizing the Adomian decomposition method, followed by coupling some numerical integration schemes or quadrature rules. These quadrature rules are used to approximate the definite integrals which are analytically impossible [23] [24] [25] [26] as the solution is given at the nodes. For convenience, the proposed extension is known as Laplace Discrete Adomian Decomposition Method (LDADM). The rest of this paper is arranged as follows. Section 2 describes the proposed LDADM. Section 3 presents numerical results with four examples and finally Section 4 concludes.

\section{Laplace Discrete Adomian Decomposition Method}

In this section, we present the Laplace discrete Adomian decomposition method over the integro-differential equation of the form

$$
u^{\prime \prime}(x)=f(x)+\int_{a}^{b} k(x, t) \cdot(R u(t)+N(u(t))) \mathrm{d} t,
$$

Subject to the following initial conditions

$$
u(0)=\alpha, u^{\prime}(0)=\beta .
$$

To solve the nonlinear integro-differential Equation (2.1) using the Laplace transform method, we recall the Laplace transform of the second derivative of $u(x)$, that is $\mathcal{L}\left\{u^{\prime \prime}(x)\right\}=s^{2} \mathcal{L}\{u(x)\}-s u(0)-u^{\prime}(0)$. Thus on applying the Laplace transform to both sides of Equation (2.1) we obtain

$$
\begin{aligned}
& s^{2} \mathcal{L}\{u(x)\}-s u(0)-u^{\prime}(0) \\
& =\mathcal{L}\{f(x)\}+\mathcal{L}\left\{\int_{a}^{b} k(x, t) \cdot(R u(t)+N(u(t))) \mathrm{d} t\right\}
\end{aligned}
$$

We thus obtain the following equation after using the above prescribed initial conditions 


$$
\mathcal{L}\{u(x)\}=\frac{\alpha}{s}+\frac{\beta}{s^{2}}+\frac{1}{s^{2}} \mathcal{L}\{f(x)\}+\frac{1}{s^{2}} \mathcal{L} \int_{a}^{b} k(x, t)(R u(t)+N(u(t))) \mathrm{d} t .
$$

The decomposition method represents the solution $u(x)$ as a series of the form:

$$
u(x)=\sum_{n=0}^{\infty} u_{n}(x),
$$

and the nonlinear term $\mathrm{Nu}(t)$ is decomposed into an infinite series of the form:

$$
N(u(t))=\sum_{n=0}^{\infty} A_{n},
$$

where $A_{n}$ 's are the Adomian polynomials of $u_{0}, u_{1}, \cdots, u_{n}$ given by the formula

$$
A_{n}=\frac{1}{n !} \frac{\mathrm{d}^{n}}{\mathrm{~d} \lambda^{n}}\left[N\left(\sum_{i=0}^{n} \lambda^{i} u_{i}\right)\right]_{\lambda=0}, i=0,1,2, \cdots
$$

On substituting Equation's (2.4) and (2.5) in Equation (2.3) and making comparison between the right and left hand sides, we thus obtain:

$$
\left\{\begin{array}{l}
\mathcal{L}\left\{u_{0}(x)\right\}=\frac{\alpha}{s}+\frac{\beta}{s^{2}}+\frac{1}{s^{2}} \mathcal{L}(f(x)) \\
\mathcal{L}\left\{u_{k+1}(x)\right\}=\frac{1}{s^{2}} \mathcal{L} \int_{a}^{b} k(x, t)\left(R u_{k}(x)+A_{k}(x)\right), k \geq 0 .
\end{array}\right.
$$

Furthermore, if the evaluation of the integrals in Equation (2.7) is analytically possible, the ADM can be applied directly. However, in the case where the evaluation of integrals is analytically impossible we consider the numerical integration scheme given by the following formula:

$$
\int_{a}^{b} f(t) \mathrm{d} t \approx \sum_{j=0}^{0} w_{n, i} f\left(t_{n, i}\right),
$$

where $f(t)$ is continuous function on $[a, b], t_{n, i}=a+i h$ are the nodes of the numerical integration, $h=\frac{b-a}{n}$ is the fixed step length and $w_{n, i}, i=0,1,2, \cdots, n$ are the weights functions. Now, applying the formula given in Equation (2.8) on Equation (2.7) to obtain

$$
\left\{\begin{array}{l}
\mathcal{L}\left\{u_{0}(x)\right\}=\frac{\alpha}{s}+\frac{\beta}{s^{2}}+\frac{1}{s^{2}} \mathcal{L}(f(x)) \\
\mathcal{L}\left\{u_{k+1}(x)\right\}=\frac{1}{s^{2}} \mathcal{L}\left(\sum_{i=0}^{n} w_{n, i} k\left(x, t_{n, i}\right) \cdot\left(R\left(u_{k}\left(t_{n, i}\right)\right)+A_{k}\left(t_{n, i}\right)\right)\right), k \geq 0 .
\end{array}\right.
$$

Finally, on applying the inverse Laplace transform to the first part of Equation (2.9) gives $u_{0}(x)$, and, consequently will define $A_{0}$. Also, using $A_{0}$ enables us to evaluate $u_{1}(x)$. The determination of $u_{0}(x)$ and $u_{1}(x)$ leads to the determination of $A_{1}$ that will allow us to determine $u_{2}(x)$, and so on. This in turn will lead to the complete determination of the components of $u_{k}, k \geq 0$ upon using the second part of Equation (2.9).

$$
\left\{\begin{array}{l}
u_{0}(x)=\mathcal{L}^{-1}\left\{\frac{\alpha}{s}+\frac{\beta}{s^{2}}+\frac{1}{s^{2}} \mathcal{L}(f(x))\right\} \\
u_{k+1}(x)=\mathcal{L}^{-1}\left\{\frac{1}{s^{2}} \mathcal{L}\left(\sum_{i=0}^{n} w_{n, i} k\left(x, t_{n, i}\right) \cdot\left(R\left(u_{k}\left(t_{n, i}\right)\right)+A_{k}\left(t_{n, i}\right)\right)\right)\right\}, k \geq 0
\end{array}\right.
$$


From this recursive relation in Equation (2.10), $u_{0}, u_{1}, u_{2}, \cdots$ can be calculated. The solution of Equation (2.1) is now determined in Equation (2.10). However, in practice the series $\sum_{n=0}^{\infty} u_{n}$ must be truncated to the series:

$$
\varphi_{n}=\sum_{i=0}^{n} u_{i} \text { with } \lim _{n \rightarrow \infty} \varphi_{n}=u
$$

\subsection{Trapezoidal Method (TR)}

We couple the trapezoidal method (TR) to Equation (2.10) to obtain:

$$
\left\{\begin{aligned}
u_{0}(x)=\alpha & +\beta x+L^{-1} f(x) \\
u_{k+1}(x)= & L^{-1}\left(\frac { h } { 2 } \left(k(x, a)\left(R\left(u_{k}(a)\right)+A_{k}(a)\right)\right.\right. \\
& +2 \sum_{i=1}^{n-1} k\left(x, x_{i}\right)\left(R\left(u_{k}\left(x_{i}\right)\right)+A_{k}\left(x_{i}\right)\right) \\
& \left.+k(x, b)\left(R\left(u_{k}(b)\right)+A_{k}(b)\right)\right), k \geq 0 .
\end{aligned}\right.
$$

\subsection{Simpson's Method (SM)}

Also, on coupling the Simpson's method (SM) to Equation (2.10), we get:

$$
\left\{\begin{aligned}
u_{0}(x)=\alpha & +\beta x+L^{-1} f(x) \\
u_{k+1}(x)= & L^{-1}\left(\frac { h } { 2 } \left(k(x, a)\left(R\left(u_{k}(a)\right)+A_{k}(a)\right)\right.\right. \\
& +4 \sum_{i=1}^{\frac{n}{2}} k\left(x, x_{2 i-1}\right)\left(R\left(u\left(x_{2 i-1}\right)\right)+A_{k}\left(x_{2 i-1}\right)\right) \\
& +2 \sum_{i=1}^{\frac{n}{2}-1} k\left(x, x_{2 i}\right)\left(R\left(u\left(x_{2 i}\right)\right)+A_{k}\left(x_{2 i}\right)\right) \\
& \left.+k(x, b)\left(R\left(u_{k}(b)\right)+A_{k}(b)\right)\right), k \geq 0 .
\end{aligned}\right.
$$

\section{Computational Results and Analysis}

In this section, we consider several nonlinear integro-differential equations as examples in order to show the efficiency and the simplicity of the proposed method. We start with the nonlinear Volterra and Fredholm integro-differential equations down to their systems, respectively.

\section{Example 3.1}

Consider the nonlinear Volterra integro-differential equation

$$
u^{\prime}(x)=\frac{1}{5} x^{5}-\int_{0}^{x}\left(u^{2}(t)-2\right) \mathrm{d} t, u(0)=0,
$$

with the exact solution given by $u(x)=x^{2}$.

In order to use the quadrature rule for Equation (3.1), let $t=x \cdot v$, we get

$$
u^{\prime}(x)=\frac{1}{5} x^{5}-x \int_{0}^{1}\left(u^{2}(x \cdot v)-2\right) \mathrm{d} v, u(0)=0 .
$$

Taking the Laplace transform of both sides of the above equation gives

$$
\mathcal{L}\left\{u^{\prime}(x)\right\}=\mathcal{L}\left(\frac{1}{5} x^{5}\right)-\mathcal{L}\left(x \int_{0}^{1}\left(u^{2}(x \cdot v)-2\right) \mathrm{d} v\right) .
$$


So that,

$$
s \mathcal{L}\{u(x)\}-u(0)=\frac{24}{s^{6}}-\mathcal{L}\left(x \int_{0}^{1}\left(u^{2}(x \cdot v)-2\right) \mathrm{d} v\right),
$$

or equivalently

$$
\mathcal{L}\{u(x)\}=\frac{24}{s^{7}}-\frac{1}{s} \mathcal{L}\left(x \int_{0}^{1}\left(u^{2}(x \cdot v)-2\right) \mathrm{d} v\right) .
$$

1) Trapezoidal Method (TM)

We divide the interval $(0,1)$ into subinterval of equal lengths $h=0.2, n=5$ and denote $v_{i}=a+i h, 0 \leq i \leq 5$.

The recursive relation is given by

$$
\left\{\begin{array}{l}
\mathcal{L}\left\{u_{0}(x)\right\}=\frac{24}{s^{7}} \\
\mathcal{L}\left\{u_{k+1}(x)\right\}=-\frac{0.1}{s} \mathcal{L}\left(x \cdot\left(x A_{k}\left(v_{0}\right)+2 \sum_{i=1}^{4} x A_{k}\left(v_{i}\right)+x A_{k}\left(v_{5}\right)\right)\right), k \geq 0
\end{array}\right.
$$

or

$$
\left\{\begin{aligned}
\mathcal{L}\left\{u_{1}(x)\right\} & =\frac{2}{s^{3}}-\frac{7.900194170}{s^{15}} \\
\mathcal{L}\left\{u_{2}(x)\right\} & =-\frac{3315.398639}{s^{11}}-\frac{3.15801038}{s^{23}} \\
& \vdots
\end{aligned}\right.
$$

Taking the inverse Laplace transform of both sides of the first part of recursive relation, and using this recursive relation gives

$$
\left\{\begin{array}{l}
u_{0}(x)=\frac{1}{30} x^{6} \\
u_{1}(x)=x^{2}-0.00000906 x^{14} \\
\vdots
\end{array}\right.
$$

The series solution is obtained by summing the following iterates

$$
u(x)=u_{0}+u_{1}+u_{2}+\cdots .
$$

The results produced by the present method with only few components $(m=$ $5)$ are in a very good agreement with the exact solution results as shown in Table 1 and illustrated graphically in Figure 1.

\section{2) Simpson's Method (SM)}

We divide the interval $(0,1)$ into subinterval of equal lengths $h=0.1, n=10$ and denote $x_{i}=a+i h, 0 \leq i \leq 10$. The recursive relation is given by

$$
\left\{\begin{aligned}
\mathcal{L}\left\{u_{0}(x)\right\}= & \frac{24}{s^{7}}, \\
\mathcal{L}\left\{u_{k+1}(x)\right\}= & -\frac{0.1}{3 s} \mathcal{L}\left(x A_{k}\left(v_{0}\right)+4 \sum_{i=1}^{5} x A_{k}\left(v_{2 i-1}\right)\right. \\
& \left.+2 \sum_{i=1}^{4} x A_{k}\left(v_{2 i}\right)+x A_{k}\left(v_{10}\right)\right), k \geq 0,
\end{aligned}\right.
$$


Table 1. Comparison between the exact solution $u(x)$ and approximate solution using LDADM based on TM in EX. 3.1.

\begin{tabular}{cccc}
\hline $\boldsymbol{x}$ & Exact & LDADM & Absolute Error \\
\hline 0 & 0 & 0 & 0 \\
0.20 & 0.04000000 & 0.03999986 & $1.41640000 \mathrm{e}-07$ \\
0.40 & 0.16000000 & 0.15999094 & $9.05930000 \mathrm{e}-06$ \\
0.60 & 0.36000000 & 0.35989712 & $1.02878600 \mathrm{e}-04$ \\
0.80 & 0.64000000 & 0.63942742 & $5.72582800 \mathrm{e}-04$ \\
1 & 1.00000000 & 0.99787295 & $2.12705460 \mathrm{e}-03$ \\
\hline
\end{tabular}

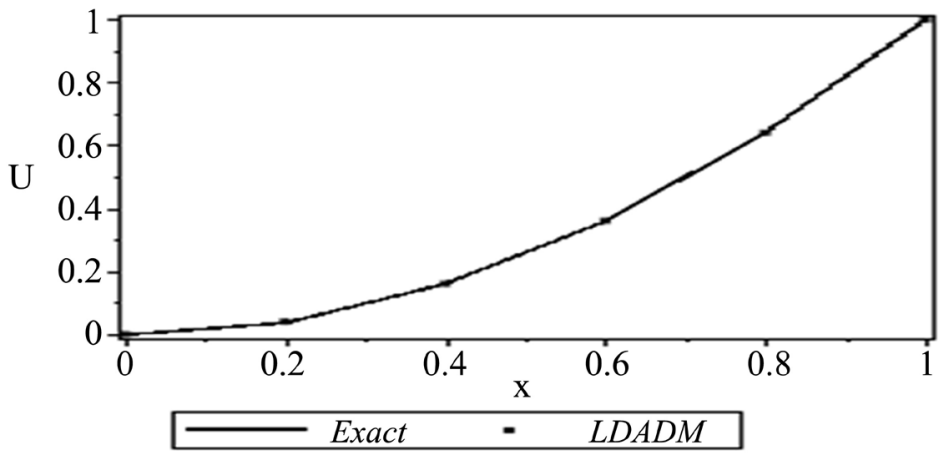

Figure 1. Curves of the exact solution $u(x)$ and approximate solution using LDADM based on TM in EX. 3.1.

Or

$$
\left\{\begin{aligned}
\mathcal{L}\left\{u_{1}(x)\right\} & =\frac{2}{s^{3}}-\frac{5.36881755}{s^{15}} \\
\mathcal{L}\left\{u_{2}(x)\right\} & =-\frac{2692.40899737}{s^{11}}+\frac{1.05763414}{s^{23}}, \\
& \vdots
\end{aligned}\right.
$$

Taking the inverse Laplace transform of both sides of the first part of recursive relation, and using this recursive relation gives

$$
\left\{\begin{aligned}
& u_{0}(x)=\frac{1}{30} x^{6} \\
& u_{1}(x)=x^{2}-0.00000616 x^{14} \\
& \vdots
\end{aligned}\right.
$$

The series solution is obtained by summing the following iterates

$$
u(x)=u_{0}+u_{1}+u_{2}+\cdots .
$$

The results produced by the present method with only few components $(m=$ 5 ) are in a very good agreement with the exact solution results as shown in Table 2 and illustrated graphically in Figure 2.

Example 3.2

Consider the nonlinear Fredholm integro-differential equation 
Table 2. Comparison between the exact solution $u(x)$ and approximate solution using LDADM based on SM in EX. 3.1.

\begin{tabular}{cccc}
\hline $\boldsymbol{x}$ & Exact & LDADM & Absolute Error \\
\hline 0 & 0 & 0 & 0 \\
0.10 & 0.01000000 & 0.01000000 & $2.22221713 \mathrm{e}-12$ \\
0.20 & 0.04000000 & 0.04000000 & $1.42214800 \mathrm{e}-10$ \\
0.30 & 0.09000000 & 0.09000000 & $1.61901986 \mathrm{e}-09$ \\
0.40 & 0.16000000 & 0.15999999 & $9.05840967 \mathrm{e}-09$ \\
0.50 & 0.25000000 & 0.24999997 & $3.37952007 \mathrm{e}-08$ \\
\hline
\end{tabular}

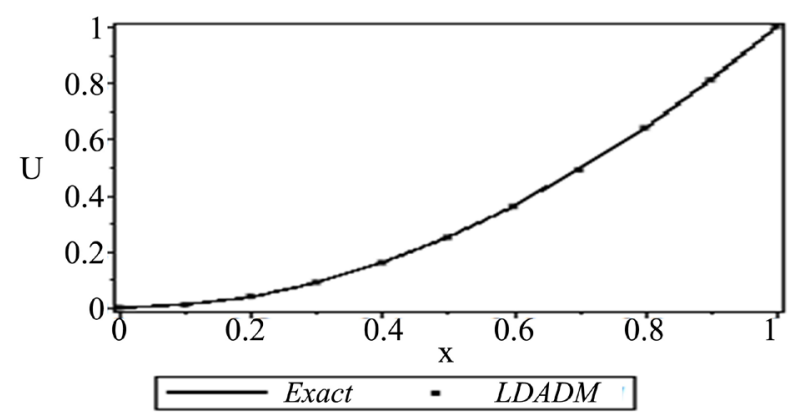

Figure 2. Curves of the exact solution $u(x)$ and approximate solution using LDADM based on SM in EX. 3.1.

$$
u^{\prime \prime}(x)=\frac{1}{2} \mathrm{e}^{x}+\frac{1}{2} \int_{0}^{1} \mathrm{e}^{x-2 t} \cdot u^{2}(t) \mathrm{d} t, u^{\prime}(0)=u(0)=1,
$$

with the exact solution is $u(x)=\mathrm{e}^{x}$.

Taking the Laplace transform of both sides of the given equation gives

$$
\mathcal{L}\left\{u^{\prime \prime}(x)\right\}=\mathcal{L}\left(\frac{1}{2} \mathrm{e}^{x}\right)+\frac{1}{2} \mathcal{L}\left(\int_{0}^{1} \mathrm{e}^{x-2 t} \cdot u^{2}(t) \mathrm{d} t\right)
$$

So that

$$
s^{2} \mathcal{L}\{u(x)\}-s u(0)-u^{\prime}(0)=\frac{1}{2(s-1)}+\frac{1}{2} \mathcal{L}\left(\int_{0}^{1} \mathrm{e}^{x-2 t} \cdot u^{2}(t) \mathrm{d} t\right)
$$

or equivalently

$$
\mathcal{L}\{u(x)\}=\frac{1}{s}+\frac{1}{s^{2}}+\frac{1}{2 s^{2}(s-1)}+\frac{1}{2 s^{2}} \mathcal{L}\left(\int_{0}^{1} \mathrm{e}^{x-2 t} \cdot u^{2}(t) \mathrm{d} t\right) .
$$

\section{1) Trapezoidal Method (TM)}

Let $h=0.2, n=5$, the recursive relation is given by

$$
\left\{\begin{aligned}
\mathcal{L}\left\{u_{0}(x)\right\}= & \frac{1}{s}+\frac{1}{s^{2}}+\frac{1}{2 s^{2}(s-1)} \\
\mathcal{L}\left\{u_{k+1}(x)\right\}= & \frac{0.2}{4 s^{2}} \mathcal{L}\left(\mathrm{e}^{x-2 t_{0}} \cdot A_{k}\left(t_{0}\right)+2 \sum_{i=1}^{4} \mathrm{e}^{x-2 t_{i}} \cdot A_{k}\left(t_{i}\right)\right. \\
& \left.+\mathrm{e}^{x-2 t_{5}} \cdot A_{k}\left(t_{5}\right)\right), k \geq 0
\end{aligned}\right.
$$


Taking the inverse Laplace transform of both sides of the first part of recursive relation, and using this recursive relation gives

$$
\left\{\begin{aligned}
u_{0}(x) & =\frac{1}{2}+\frac{1}{2} x+\frac{1}{2} \mathrm{e}^{x}, \\
u_{1}(x) & =-0.44983021-0.44983021 x+0.44983021 \mathrm{e}^{x}, \\
& \vdots
\end{aligned}\right.
$$

The series solution is obtained by summing the following iterates

$$
u(x)=u_{0}+u_{1}+u_{2}+\cdots .
$$

The results produced by the present method with only few components $(m=$ 5 ) are in a very good agreement with the exact solution results as shown in Table 3 and illustrated graphically in Figure 3.

\section{2) Simpson's Method (SM)}

Let $h=0.1, n=10$, the recursive relation is given by

$$
\left\{\begin{aligned}
\mathcal{L}\left\{u_{0}(x)\right\}= & \frac{1}{s}+\frac{1}{s^{2}}+\frac{1}{2 s^{2}(s-1)}, \\
\mathcal{L}\left\{u_{k+1}(x)\right\}= & \frac{0.1}{6 s^{2}} \mathcal{L}\left(\mathrm{e}^{x-2 t_{0}} \cdot A_{k}\left(t_{0}\right)+4 \sum_{i=1}^{5} \mathrm{e}^{x-2 t_{2 i-1}} \cdot A_{k}\left(t_{2 i-1}\right)\right. \\
& \left.+2 \sum_{i=1}^{2} \mathrm{e}^{x-2 t_{2 i}} \cdot A_{k}\left(t_{2 i}\right)+\mathrm{e}^{x-2 t_{5}} \cdot A_{k}\left(t_{5}\right)\right), k \geq 0 .
\end{aligned}\right.
$$

Table 3. Comparison between the exact solution $u(x)$ and Approximate solution using LDADM based on RM in EX. 3.2.

\begin{tabular}{cccc}
\hline $\boldsymbol{x}$ & Exact & DADM & Absolute Error \\
\hline 0 & 1.00000000 & 1.00000000 & 0 \\
0.20 & 1.22140276 & 1.22140206 & $7.03000000 \mathrm{e}-07$ \\
0.40 & 1.49182470 & 1.49182168 & $3.01500000 \mathrm{e}-06$ \\
0.60 & 1.82211880 & 1.82211150 & $7.29500000 \mathrm{e}-06$ \\
0.80 & 2.22554093 & 2.22552695 & $1.39750000 \mathrm{e}-05$ \\
1 & 2.71828183 & 2.71825824 & $2.35900000 \mathrm{e}-05$ \\
\hline
\end{tabular}

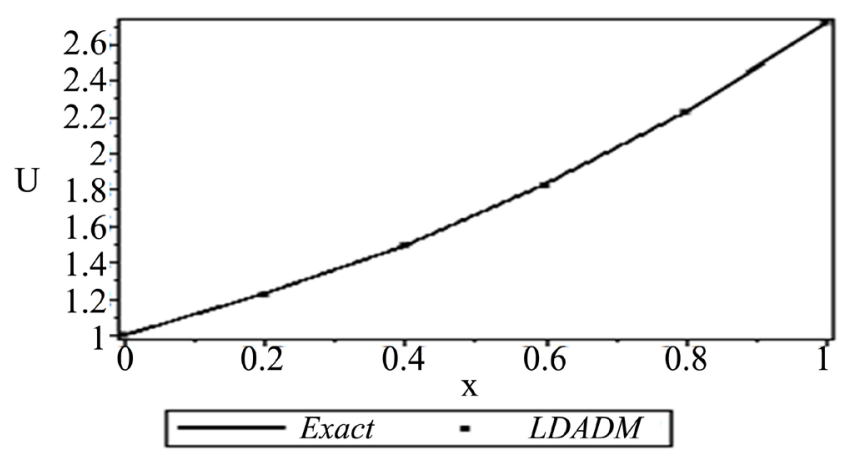

Figure 3. Curves of the exact solution $u(x)$ and approximate solution using LDADM based on TM in EX. 3.2. 
Taking the inverse Laplace transform of both sides of the first part of recursive relation, and using this recursive relation gives

$$
\left\{\begin{aligned}
u_{0}(x) & =\frac{1}{2}+\frac{1}{2} x+\frac{1}{2} \mathrm{e}^{x}, \\
u_{1}(x) & =-0.45035999-0.45035999 x+0.450359991 \mathrm{e}^{x}, \\
& \vdots
\end{aligned}\right.
$$

The series solution is obtained by summing the following iterates

$$
u(x)=u_{0}+u_{1}+u_{2}+\cdots .
$$

The results produced by the present method with only few components $(m=$ 5 ) are in a very good agreement with the exact solution results as shown in Table 4 and illustrated graphically in Figure 4.

Example 3.3

Consider the system of nonlinear Volterra integro differential equation

$$
\left\{\begin{array}{l}
u^{\prime}(x)=3 x^{2}-\frac{2}{3} x^{3}-\frac{1}{126} x^{9}+\int_{0}^{x}(x-t)^{2}\left(u^{2}(t)+v^{2}(t)\right) \mathrm{d} t, u(0)=1, \\
v^{\prime}(x)=-3 x^{2}-\frac{1}{35} x^{7}+\int_{0}^{x}(x-t)^{2}\left(u^{2}(t)-v^{2}(t)\right) \mathrm{d} t, v(0)=1,
\end{array}\right.
$$

with the exact solution $(u(x), v(x))=\left(1+x^{3}, 1-x^{3}\right)$.

In order to use the quadrature rule for Equation (3.2), let $t=x \cdot v$, we get

Table 4. Comparison between the exact solution $u(x)$ and approximate solution using LDADM based on SM in EX. 3.2.

\begin{tabular}{cccc}
\hline$x$ & Exact & DADM & Absolute Error \\
\hline 0 & 1.00000000 & 1.00000000 & 0 \\
0.10 & 1.10517092 & 1.10517076 & $1.58713332 \mathrm{e}-07$ \\
0.20 & 1.22140276 & 1.22140210 & $6.56924555 \mathrm{e}-07$ \\
0.30 & 1.34985881 & 1.34985728 & $1.53033898 \mathrm{e}-06$ \\
0.40 & 1.49182470 & 1.49182188 & $2.81841706 \mathrm{e}-06$ \\
0.50 & 1.64872127 & 1.64871671 & $4.56476936 \mathrm{e}-06$ \\
\hline
\end{tabular}



Figure 4. Curves of the exact solution $u(x)$ and approximate solution using LDADM based on SM in EX. 3.2. 


$$
\left\{\begin{array}{l}
u^{\prime}(x)=3 x^{2}-\frac{2}{3} x^{3}-\frac{1}{126} x^{9}+x \int_{0}^{1}(x-x \cdot v)^{2}\left(u^{2}(x \cdot v)+v^{2}(x \cdot v)\right) \mathrm{d} v, u(0)=1, \\
v^{\prime}(x)=-3 x^{2}-\frac{1}{35} x^{7}+x \int_{0}^{1}(x-x \cdot v)^{2}\left(u^{2}(x \cdot v)-v^{2}(x \cdot v)\right) \mathrm{d} v, v(0)=1 .
\end{array}\right.
$$

Taking the Laplace transform of both sides of the above equation gives

$$
\left\{\begin{array}{l}
s \mathcal{L}\{u(x)\}-1=\mathcal{L}\left(3 x^{2}-\frac{2}{3} x^{3}-\frac{1}{126} x^{9}\right)+\mathcal{L}\left(x \int_{0}^{1}(x-x \cdot v)^{2}\left(u^{2}(x \cdot v)+v^{2}(x \cdot v)\right) \mathrm{d} v\right), \\
s \mathcal{L}\{v(x)\}-1=\mathcal{L}\left(-3 x^{2}-\frac{1}{35} x^{7}\right)+\mathcal{L}\left(x \int_{0}^{1}(x-x \cdot v)^{2}\left(u^{2}(x \cdot v)-v^{2}(x \cdot v)\right) \mathrm{d} v\right) .
\end{array}\right.
$$

So that

$$
\left\{\begin{array}{l}
\mathcal{L}\{u(x)\}=\frac{1}{s}+\frac{6}{s^{4}}-\frac{4}{s^{5}}-\frac{2880}{s^{11}}+\frac{1}{s} \mathcal{L}\left(x \int_{0}^{1}(x-x \cdot v)^{2}\left(u^{2}(x \cdot v)+v^{2}(x \cdot v)\right) \mathrm{d} v\right), \\
\mathcal{L}\{v(x)\}=\frac{1}{s}-\frac{6}{s^{4}}-\frac{144}{s^{9}}+\frac{1}{s} \mathcal{L}\left(x \int_{0}^{1}(x-x \cdot v)^{2}\left(u^{2}(x \cdot v)-v^{2}(x \cdot v)\right) \mathrm{d} v\right) .
\end{array}\right.
$$

\section{1) Trapezoidal Method (TM)}

We divide the interval $(0,1)$ into subinterval of equal lengths $h=0.2, n=5$ and denote $v_{i}=a+i h, 0 \leq i \leq 5$. The recursive relations as

$$
\begin{gathered}
\left\{\begin{aligned}
\mathcal{L}\left\{u_{0}(x)\right\}=\frac{1}{s}+\frac{6}{s^{4}}-\frac{4}{s^{5}}-\frac{2880}{s^{11}}, \\
\mathcal{L}\left\{v_{0}(x)\right\}=\frac{1}{s}-\frac{6}{s^{4}}-\frac{144}{s^{9}},
\end{aligned}\right. \\
\left\{\begin{aligned}
\mathcal{L}\left(u_{k+1}(x)\right)= & \frac{1}{s} \mathcal{L}\left(x\left(\left(x-x \cdot v_{0}\right)^{2}\left(A_{k}\left(v_{0}\right)+B_{k}\left(v_{0}\right)\right)\right)\right. \\
& +2 \sum_{i=1}^{4}\left(x-x \cdot v_{i}\right)^{2}\left(A_{k}\left(v_{i}\right)+B_{k}\left(v_{i}\right)\right) \\
& \left.+\left(x-x \cdot v_{5}\right)^{2}\left(A_{k}\left(v_{5}\right)+B_{k}\left(v_{5}\right)\right)\right), k \geq 0 \\
\mathcal{L}\left(v_{k+1}(x)\right)= & \frac{1}{s} \mathcal{L}\left(x\left(\left(x-x \cdot v_{0}\right)^{2}\left(A_{k}\left(v_{0}\right)-B_{k}\left(v_{0}\right)\right)\right)\right. \\
& +2 \sum_{i=1}^{4}\left(x-x \cdot v_{i}\right)^{2}\left(A_{k}\left(v_{i}\right)-B_{k}\left(v_{i}\right)\right) \\
& \left.+\left(x-x \cdot v_{5}\right)^{2}\left(A_{k}\left(v_{5}\right)-B_{k}\left(v_{5}\right)\right)\right), k \geq 0
\end{aligned}\right.
\end{gathered}
$$

Taking the inverse Laplace transform of both sides of the first part of recursive relation, and using this recursive relation gives

$$
\left\{\begin{array}{l}
u_{0}=1+x^{3}-\frac{1}{6} x^{4}-\frac{1}{1260} x^{10} \\
v_{0}=1-x^{3}-\frac{1}{280} x^{8} \\
u_{1}=0.17 x^{4}-0.00039467 x^{8} \\
v_{1}=0.00950857 x^{7}-0.00039467 x^{8} \\
\quad \vdots
\end{array}\right.
$$

The series solutions are

$$
\left\{\begin{array}{l}
u(x)=u_{0}+u_{1}+u_{2}+\cdots \\
v(x)=v_{0}+v_{1}+v_{2}+\cdots
\end{array}\right.
$$


The results produced by the method with only few components $(m=5)$ are in a very good agreement with the exact solution results as shown in Table 5 and Table 6 and illustrated graphically given in Figure 5 and Figure 6.

\section{2) Simpson's Method (SM)}

We divide the interval $(0,1)$ into subinterval with equal length $h=0.1, n=10$ and denote $x_{i}=a+i h, 0 \leq i \leq 10$. The recursive relation is given by

$$
\begin{aligned}
& \left\{\begin{array}{l}
\mathcal{L}\left\{u_{0}(x)\right\}=\frac{1}{s}+\frac{6}{s^{4}}-\frac{4}{s^{5}}-\frac{2880}{s^{11}}, \\
\mathcal{L}\left\{v_{0}(x)\right\}=\frac{1}{s}-\frac{6}{s^{4}}-\frac{144}{s^{9}},
\end{array}\right. \\
& \mathcal{L}\left(u_{k+1}(x)\right)=\frac{0.1}{3 s} \mathcal{L}\left(x\left(\left(x-x \cdot v_{0}\right)^{2}\left(A_{k}\left(v_{0}\right)+B_{k}\left(v_{0}\right)\right)\right)\right. \\
& +4 \sum_{i=1}^{4}\left(x-x \cdot v_{2 i-1}\right)^{2}\left(A_{k}\left(v_{2 i-1}\right)+B_{k}\left(v_{2 i-1}\right)\right) \\
& +2 \sum_{i=1}^{4}\left(x-x \cdot v_{2 i}\right)^{2}\left(A_{k}\left(v_{2 i}\right)+B_{k}\left(v_{2 i}\right)\right) \\
& \left.+\left(x-x \cdot v_{10}\right)^{2}\left(A_{k}\left(v_{10}\right)+B_{k}\left(v_{10}\right)\right)\right), k \geq 0 \\
& \mathcal{L}\left(v_{k+1}(x)\right)=\frac{0.1}{3 s} \mathcal{L}\left(x\left(\left(x-x \cdot v_{0}\right)^{2}\left(A_{k}\left(v_{0}\right)-B_{k}\left(v_{0}\right)\right)\right)\right. \\
& +4 \sum_{i=1}^{4}\left(x-x \cdot v_{2 i-1}\right)^{2}\left(A_{k}\left(v_{2 i-1}\right)-B_{k}\left(v_{2 i-1}\right)\right) \\
& +2 \sum_{i=1}^{4}\left(x-x \cdot v_{2 i}\right)^{2}\left(A_{k}\left(v_{2 i}\right)-B_{k}\left(v_{2 i}\right)\right) \\
& \left.+\left(x-x \cdot v_{10}\right)^{2}\left(A_{k}\left(v_{10}\right)-B_{k}\left(v_{10}\right)\right)\right), k \geq 0
\end{aligned}
$$

Taking the inverse Laplace transform of both sides of the first part of recursive relation, and using this recursive relation gives

$$
\left\{\begin{array}{l}
u_{0}=1+x^{3}-\frac{1}{6} x^{4}-\frac{1}{1260} x^{10}, \\
v_{0}=1-x^{3}-\frac{1}{280} x^{8}, \\
u_{1}=0.16666667 x^{4}-0.00039736 x^{8}, \\
v_{1}=0.00952761 x^{7}-0.00039736 x^{8}, \\
\quad \vdots
\end{array}\right.
$$

Table 5. Comparison between the exact solution $u(x)$ and approximate solution using LDADM based on TM in EX. 3.3.

\begin{tabular}{cccc}
\hline$x$ & Exact & DADM & Absolute Error \\
\hline 0 & 1.00000000 & 1.00000000 & 0 \\
0.20 & 1.00800000 & 1.00800533 & $5.33335213 \mathrm{e}-06$ \\
0.40 & 1.06400000 & 1.06408534 & $8.53371745 \mathrm{e}-05$ \\
0.60 & 1.21600000 & 1.21643206 & $4.32061253 \mathrm{e}-04$ \\
0.80 & 1.51200000 & 1.51336546 & $1.36545876 \mathrm{e}-03$ \\
1 & 2.00000000 & 2.00333032 & $3.33031586 \mathrm{e}-03$ \\
\hline
\end{tabular}


Table 6. Comparison between the exact solution $v(x)$ and approximate solution using LDADM based on TM.

\begin{tabular}{cccc}
\hline$x$ & Exact & DADM & Absolute Error \\
\hline 0 & 1.00000000 & 1.00000000 & 0 \\
0.20 & 0.99200000 & 0.99200011 & $1.12587007 \mathrm{e}-07$ \\
0.40 & 0.93600000 & 0.93601324 & $1.32433409 \mathrm{e}-05$ \\
0.60 & 0.78400000 & 0.78420632 & $2.06317915 \mathrm{e}-04$ \\
0.80 & 0.48800000 & 0.48939610 & $1.39609894 \mathrm{e}-03$ \\
1 & 0.00000000 & 0.00594422 & $5.94421577 \mathrm{e}-03$ \\
\hline
\end{tabular}



Figure 5. Curves of the exact solution $u(x)$ and approximate solution using LDADM based on TM in EX. 3.3.

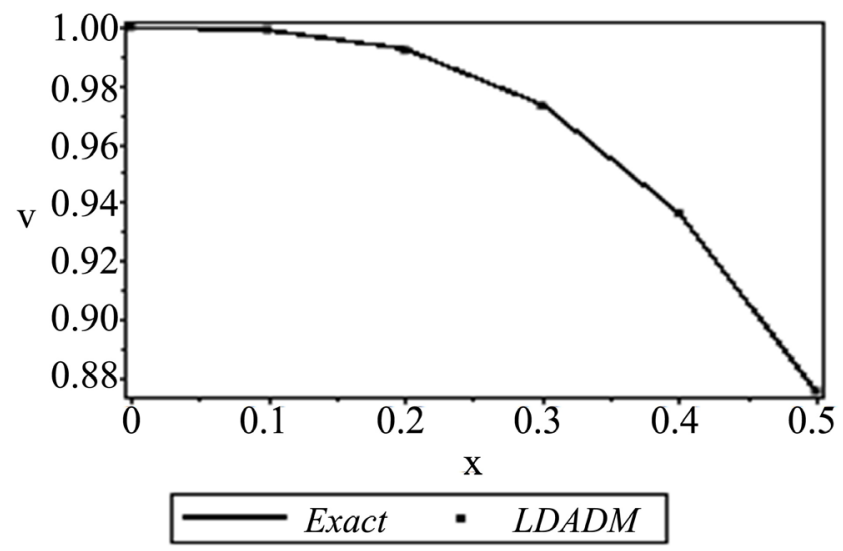

Figure 6. Curves of the exact solution $v(x)$ and approximate solution using LDADM based on TM in EX. 3.3.

The series solutions are

$$
\left\{\begin{array}{l}
u(x)=u_{0}+u_{1}+u_{2}+\cdots \\
v(x)=v_{0}+v_{1}+v_{2}+\cdots
\end{array}\right.
$$

The results produced by the method with only few components $(m=5)$ are in a very good agreement with the exact solution results as shown in Table 7 and Table 8 and illustrated graphically given in Figure 7 and Figure 8. 
Table 7. Comparison between the exact solution $u(x)$ and approximate solution using LDADM based on SM in EX. 3.3.

\begin{tabular}{cccc}
\hline $\boldsymbol{x}$ & Exact & DADM & Absolute Error \\
\hline 0 & 1.00000000 & 1.00000000 & 0 \\
0.10 & 1.00100000 & 1.00100000 & $4.16058963 \mathrm{e}-16$ \\
0.20 & 1.00800000 & 1.00800000 & $4.71714828 \mathrm{e}-13$ \\
0.30 & 1.02700000 & 1.02700000 & $2.96484081 \mathrm{e}-11$ \\
0.40 & 1.06400000 & 1.06400000 & $5.65791067 \mathrm{e}-10$ \\
0.50 & 1.12500000 & 1.12500001 & $5.58673293 \mathrm{e}-09$ \\
\hline
\end{tabular}

Table 8. Comparison between the exact solution $v(x)$ and approximate solution using LDADM based on SM in EX. 3.3.

\begin{tabular}{cccc}
\hline $\boldsymbol{x}$ & Exact & DADM & Absolute Error \\
\hline 0 & 1.00000000 & 1.00000000 & 0 \\
0.10 & 0.99900000 & 0.99900000 & $9.17047572 \mathrm{e}-10$ \\
0.20 & 0.99200000 & 0.99200011 & $1.12810573 \mathrm{e}-07$ \\
0.30 & 0.97300000 & 0.97300185 & $1.84936099 \mathrm{e}-06$ \\
0.40 & 0.93600000 & 0.93601327 & $1.32693006 \mathrm{e}-05$ \\
0.50 & 0.87500000 & 0.87506048 & $6.04816460 \mathrm{e}-05$ \\
\hline
\end{tabular}

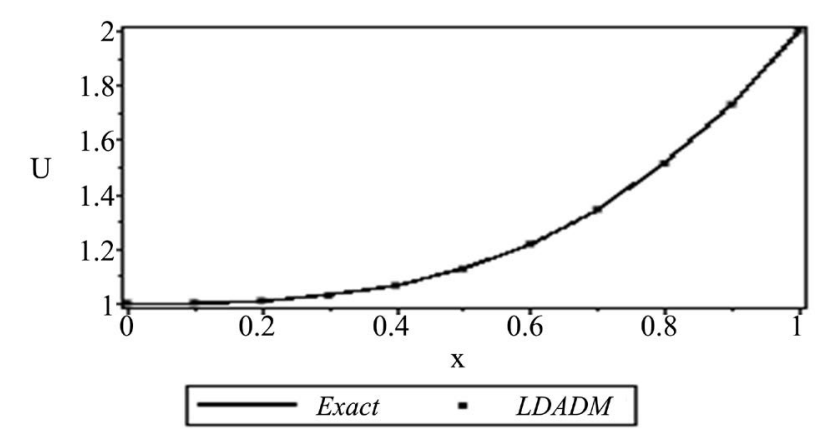

Figure 7. Curves of the exact solution $u(x)$ and approximate solution using LDADM based on SM in EX. 3.3.

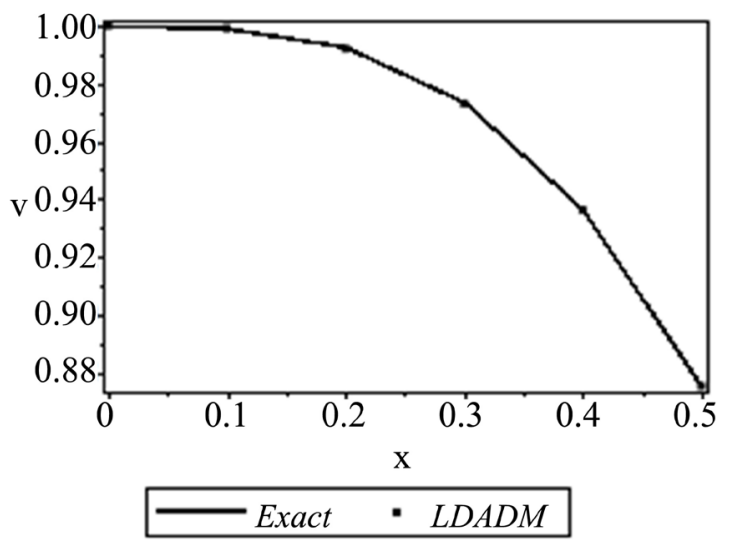

Figure 8. Curves of the exact solution $v(x)$ and approximate solution using LDADM based on SM in EX. 3.3. 


\section{Example 3.4}

Consider the system of nonlinear Fredholm integro differential equation

$$
\left\{\begin{array}{l}
u^{\prime \prime}(x)=2+\frac{12}{5} x-\int_{0}^{1} x\left(u^{2}+v^{2}\right) \mathrm{d} t, u(0)=1, u^{\prime}(0)=0, \\
v^{\prime \prime}(x)=-2+\frac{4}{3} x-\int_{0}^{1} x\left(u^{2}-v^{2}\right) \mathrm{d} t, v(0)=1, v^{\prime}(0)=0,
\end{array}\right.
$$

with the exact solution $(u(x), v(x))=\left(1+x^{2}, 1-x^{2}\right)$.

As usual, on taking the Laplace transform of both sides of the above equation gives

$$
\left\{\begin{array}{l}
s^{2} \mathcal{L}\{u(x)\}-s=\mathcal{L}\left(2+\frac{12}{5} x\right)-\mathcal{L}\left(\int_{0}^{1} x\left(u^{2}+v^{2}\right) \mathrm{d} t\right), \\
s^{2} \mathcal{L}\{v(x)\}-s=\mathcal{L}\left(-2+\frac{4}{3} x\right)-\mathcal{L}\left(\int_{0}^{1} x\left(u^{2}-v^{2}\right) \mathrm{d} t\right) .
\end{array}\right.
$$

So that

$$
\left\{\begin{array}{l}
\mathcal{L}\{u(x)\}=\frac{1}{s}+\frac{2}{s^{3}}+\frac{12}{5 s^{4}}-\frac{1}{s^{2}} \mathcal{L}\left(\int_{0}^{1} x\left(u^{2}+v^{2}\right) \mathrm{d} t\right), \\
\mathcal{L}\{u(x)\}=\frac{1}{s}-\frac{2}{s^{3}}+\frac{4}{3 s^{4}}-\frac{1}{s^{2}} \mathcal{L}\left(\int_{0}^{1} x\left(u^{2}-v^{2}\right) \mathrm{d} t\right) .
\end{array}\right.
$$

1) Trapezoidal Method (TM)

We divide the interval $(0,1)$ into subinterval of equal lengths $h=0.2, n=5$ and denote $v_{i}=a+i h, 0 \leq i \leq 5$. The recursive relations are

$$
\begin{gathered}
\left\{\begin{array}{l}
\mathcal{L}\left\{u_{0}(x)\right\}=\frac{1}{s}+\frac{2}{s^{3}}+\frac{12}{5 s^{4}}, \\
\mathcal{L}\left\{v_{0}(x)\right\}=\frac{1}{s}-\frac{2}{s^{3}}+\frac{4}{3 s^{4}} .
\end{array}\right. \\
\left\{\begin{aligned}
\mathcal{L}\left(u_{k+1}(x)\right)= & \frac{0.2}{2 s^{2}} \mathcal{L}\left(x\left(A_{k}\left(t_{0}\right)+B_{k}\left(t_{0}\right)\right)+2 \sum_{i=1}^{4}\left(A_{k}\left(v_{i}\right)+B_{k}\left(v_{i}\right)\right)\right. \\
& \left.+\left(A_{k}\left(v_{5}\right)+B_{k}\left(v_{5}\right)\right)\right), k \geq 0 \\
\mathcal{L}\left(v_{k+1}(x)\right)= & \frac{0.2}{2 s^{2}} \mathcal{L}\left(x\left(A_{k}\left(t_{0}\right)-B_{k}\left(t_{0}\right)\right)+2 \sum_{i=1}^{4}\left(A_{k}\left(v_{i}\right)-B_{k}\left(v_{i}\right)\right)\right. \\
& \left.+\left(A_{k}\left(v_{5}\right)-B_{k}\left(v_{5}\right)\right)\right), k \geq 0
\end{aligned}\right.
\end{gathered}
$$

Taking the inverse Laplace transform of both sides of the first part of recursive relation, and using this recursive relation gives

$$
\left\{\begin{array}{c}
u_{0}(x)=1+x^{2}+\frac{2}{5} x^{3}, \\
v_{0}(x)=1-x^{2}+\frac{2}{9} x^{3}, \\
u_{1}(x)=-0.47488288 x^{3}, \\
v_{1}(x)=-0.28306869 x^{3}, \\
\vdots
\end{array}\right.
$$

The series solutions are 


$$
\left\{\begin{array}{l}
u(x)=u_{0}+u_{1}+u_{2}+\cdots \\
v(x)=v_{0}+v_{1}+v_{2}+\cdots
\end{array}\right.
$$

The results produced by the method with only few components $(m=5)$ are in a very good agreement with the exact solution results as shown in Table 9 and Table 10 and illustrated graphically given in Figure 9 and Figure 10.

\section{2) Simpson's Method (SM)}

We divide the interval $(0,1)$ into subinterval of equal lengths $h=0.1, n=10$ and denote $x_{i}=a+i h, 0 \leq i \leq 10$. The recursive relation is given by

$$
\begin{gathered}
\left\{\begin{array}{l}
\mathcal{L}\left\{u_{0}(x)\right\}=\frac{1}{s}+\frac{2}{s^{3}}+\frac{12}{5 s^{4}}, \\
\mathcal{L}\left\{v_{0}(x)\right\}=\frac{1}{s}-\frac{2}{s^{3}}+\frac{4}{3 s^{4}},
\end{array}\right. \\
\left\{\begin{aligned}
\mathcal{L}\left(u_{k+1}(x)\right)= & \frac{0.1}{3 s^{2}} \mathcal{L}\left(x\left(A_{k}\left(t_{0}\right)+B_{k}\left(t_{0}\right)\right)+4 \sum_{i=1}^{5} x \cdot\left(A_{k}\left(t_{2 i-1}\right)+B_{k}\left(t_{2 i-1}\right)\right)\right. \\
& \left.+2 \sum_{i=1}^{4} x \cdot\left(A_{k}\left(t_{2 i}\right)+B_{k}\left(t_{2 i}\right)\right)+x\left(A_{k}\left(t_{10}\right)+B_{k}\left(t_{10}\right)\right)\right), k \geq 0 \\
\mathcal{L}\left(v_{k+1}(x)\right)= & \frac{0.1}{3 s^{2}} \mathcal{L}\left(x\left(A_{k}\left(t_{0}\right)-B_{k}\left(t_{0}\right)\right)+4 \sum_{i=1}^{5} x \cdot\left(A_{k}\left(t_{2 i-1}\right)-B_{k}\left(t_{2 i-1}\right)\right)\right. \\
& \left.+2 \sum_{i=1}^{4} x \cdot\left(A_{k}\left(t_{2 i}\right)-B_{k}\left(t_{2 i}\right)\right)+x\left(A_{k}\left(t_{10}\right)-B_{k}\left(t_{10}\right)\right)\right), k \geq 0
\end{aligned}\right.
\end{gathered}
$$

Taking the inverse Laplace transform of both sides of the first part of recursive relation, and using this recursive relation gives

Table 9. Comparison between the exact solution $u(x)$ and approximate solution using LDADM based on TM in EX. 3.4.

\begin{tabular}{cccc}
\hline $\boldsymbol{x}$ & Exact & DADM & Absolute Error \\
\hline 0 & 1.00000000 & 1.00000000 & 0 \\
0.20 & 1.04000000 & 1.03996370 & $3.62969667 \mathrm{e}-05$ \\
0.40 & 1.16000000 & 1.15970962 & $2.90375734 \mathrm{e}-04$ \\
0.60 & 1.36000000 & 1.35901998 & $9.80018101 \mathrm{e}-04$ \\
0.80 & 1.64000000 & 1.63767699 & $2.32300587 \mathrm{e}-03$ \\
1 & 2.00000000 & 1.99546288 & $4.53712084 \mathrm{e}-03$ \\
\hline
\end{tabular}

Table 10. Comparison between the exact solution $v(x)$ and approximate solution using LDADM based on TM in EX. 3.4.

\begin{tabular}{cccc}
\hline $\boldsymbol{x}$ & Exact & DADM & Absolute Error \\
\hline 0 & 1.00000000 & 1.00000000 & 0 \\
0.20 & 0.96000000 & 0.95996436 & $3.56392238 \mathrm{e}-05$ \\
0.40 & 0.84000000 & 0.83971489 & $2.85113790 \mathrm{e}-04$ \\
0.60 & 0.64000000 & 0.63903774 & $9.62259042 \mathrm{e}-04$ \\
0.80 & 0.36000000 & 0.35771909 & $2.28091032 \mathrm{e}-03$ \\
1 & 0 & -0.00445490 & $4.45490297 \mathrm{e}-03$ \\
\hline
\end{tabular}




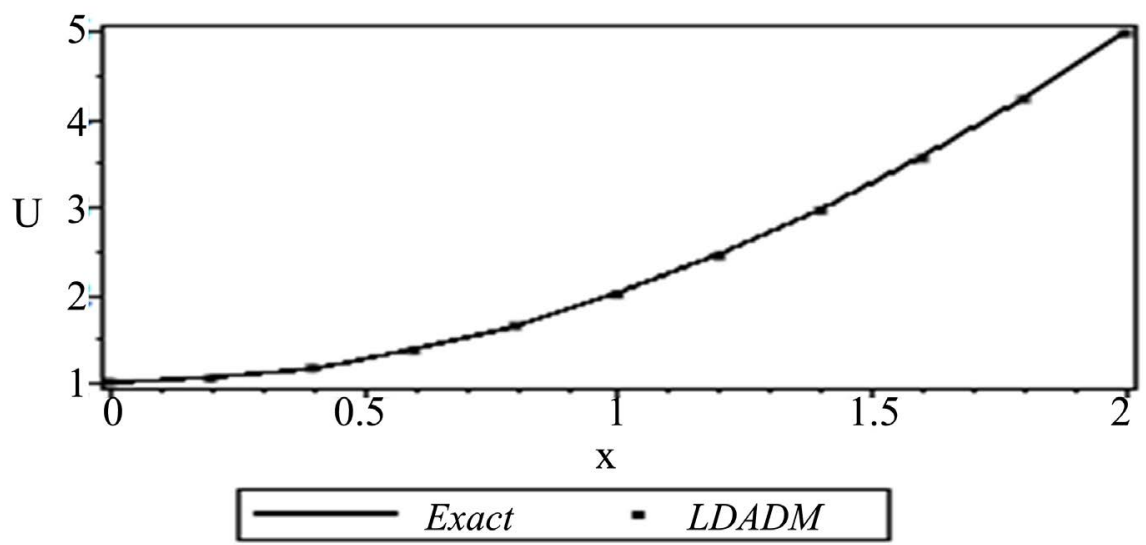

Figure 9. Curves of the exact solution $u(x)$ and approximate solution using LDADM based on TM in EX. 3.4.

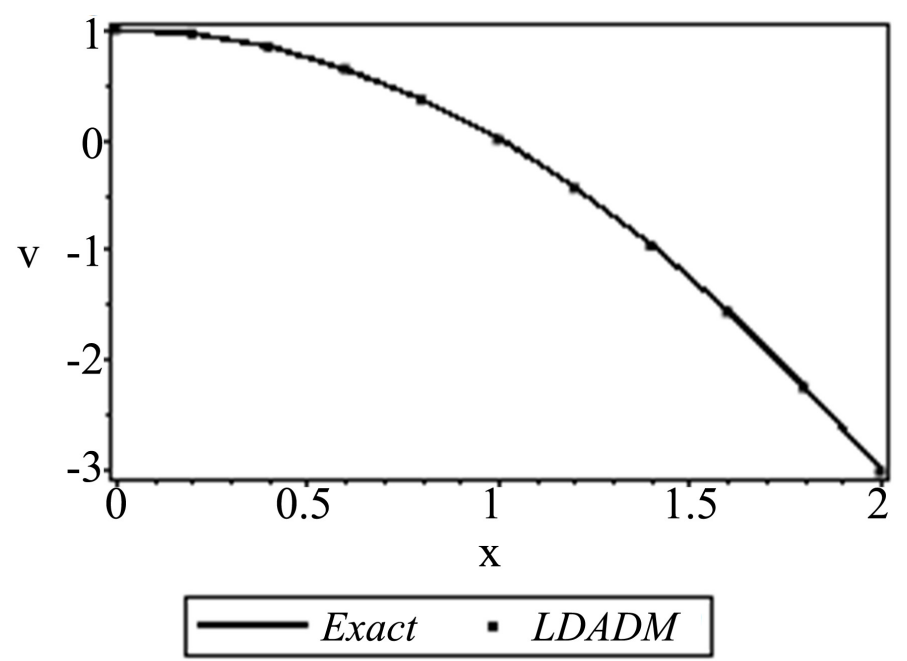

Figure 10. Curves of the exact solution $v(x)$ and approximate solution using LDADM based on TM in EX. 3.4.

$$
\left\{\begin{array}{l}
u_{0}(x)=1+x^{2}+\frac{2}{5} x^{3}, \\
v_{0}(x)=1-x^{2}+\frac{2}{9} x^{3}, \\
u_{1}(x)=-0.46672242 x^{3}, \\
v_{1}(x)=-0.27424681 x^{3}, \\
\vdots
\end{array}\right.
$$

The series solutions are

$$
\left\{\begin{array}{l}
u(x)=u_{0}+u_{1}+u_{2}+\cdots \\
v(x)=v_{0}+v_{1}+v_{2}+\cdots
\end{array}\right.
$$

The results produced by the method with only few components $(m=5)$ are in a very good agreement with the exact solution results as shown in Table 11 and Table 12 and illustrated graphically given in Figure 11 and Figure 12. 
Table 11. Comparison between the exact solution $u(x)$ and approximate solution using LDADM based on SM in EX. 3.4.

\begin{tabular}{cccc}
\hline $\boldsymbol{x}$ & Exact & DADM & Absolute Error \\
\hline 0 & 1.00000000 & 1.00000000 & 0 \\
0.10 & 1.01000000 & 1.00999945 & $5.51111213 \mathrm{e}-07$ \\
0.20 & 1.04000000 & 1.03999559 & $4.40888971 \mathrm{e}-06$ \\
0.30 & 1.09000000 & 1.08998512 & $1.48800028 \mathrm{e}-05$ \\
0.40 & 1.16000000 & 1.15996473 & $3.52711177 \mathrm{e}-05$ \\
0.50 & 1.25000000 & 1.24993111 & $6.88889017 \mathrm{e}-05$ \\
\hline
\end{tabular}

Table 12. Comparison between the exact solution $v(x)$ and approximate solution using LDADM based on SM in EX. 3.4.

\begin{tabular}{cccc}
\hline $\boldsymbol{x}$ & Exact & DADM & Absolute Error \\
\hline 0 & 1.00000000 & 1.00000000 & 0 \\
0.10 & 0.99000000 & 0.98999967 & $3.28616130 \mathrm{e}-07$ \\
0.20 & 0.96000000 & 0.95999737 & $2.62892904 \mathrm{e}-06$ \\
0.30 & 0.91000000 & 0.90999113 & $8.87263551 \mathrm{e}-06$ \\
0.40 & 0.84000000 & 0.83997897 & $2.10314323 \mathrm{e}-05$ \\
0.50 & 0.75000000 & 0.74995892 & $4.10770163 \mathrm{e}-05$ \\
\hline
\end{tabular}

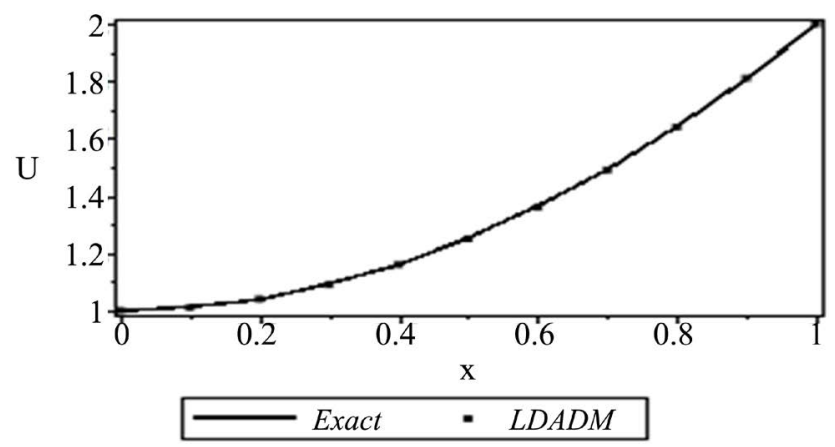

Figure 11. Curves of the exact solution $u(x)$ and approximate solution using LDADM based on SM in EX. 3.4.

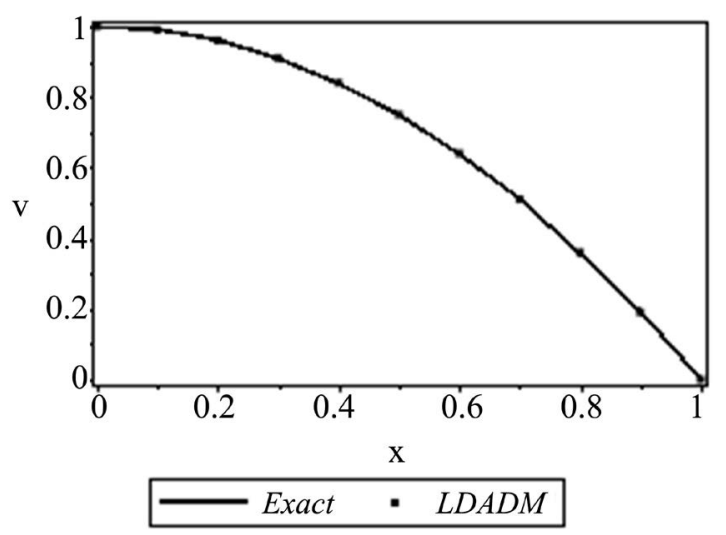

Figure 12. Curves of the exact solution $v(x)$ and approximate solution using LDADM based on SM in EX. 3.4. 


\section{Conclusion}

In this paper, a modification of the Laplace Adomian decomposition method inspired by property of discretization is proposed. We developed a new Laplace Discrete Adomian decomposition method (LDADM) in which has been successfully applied to finding efficient numerical solutions of integro-differential equations featuring both nonlinear Volterra and Fredholm integrals. The method was based on the well-known Adomian decomposition method coupled with some numerical integration schemes (quadrature rules) alongside utilizing the famous and most used Laplace transform. The method gives approximate solutions iteratively with less number of computational steps. The results reveal that the proposed method is simple to execute and effective. Thus, many highly nonlinear integro-differential equations can be solved using the proposed method.

\section{Conflicts of Interest}

The authors declare no conflicts of interest regarding the publication of this paper.

\section{References}

[1] Delves, L.M. and Mohamed, J.L. (1985) Computational Methods for Integral Equations. Cambridge University Press, Cambridge. https://doi.org/10.1017/CBO9780511569609

[2] Enright, W.H. and Hu, M. (1997) Continuous Runge-Kutta Methods for Neutral Volterra Integro-Differential Equations with Delay. Applied Numerical Mathematics, 24, 175-190. https://doi.org/10.1016/S0168-9274(97)00019-6

[3] Akyuz, A. and Sezer, M. (1999) A Chebyshev Collocation Method for the Solution of Linear Integro-Differential Equations. International Journal of Computer Mathematic, 72, 491-507. https://doi.org/10.1080/00207169908804871

[4] Karamete, A. and Sezer, M. (2002) A Taylor Collocation Method for the Solution of Linear Integro-Differential Equations. International Journal of Computer Mathematic, 79, 987-1000. https://doi.org/10.1080/00207160212116

[5] Maleknejad, K., Mirzaee, F. and Abbasbandy, S. (2004) Solving Linear Integro-Differential Equations System by Using Rationalized Haar Functions Method. Applied Mathematics and Computation, 155, 317-328. https://doi.org/10.1016/S0096-3003(03)00778-1

[6] Maleknejad, K. and Tavassoli Kajani, M. (2004) Solving Linear Integro-Differential Equation System by Galerkin Methods with Hybrid Functions. Applied Mathematics and Computation, 159, 603-612. https://doi.org/10.1016/j.amc.2003.10.046

[7] Wazwaz, A.M. (2015) A First Course in Integral Equations. World Scientific Publishing Co. Pte. Ltd., Singapore. https://doi.org/10.1142/9570

[8] Bakodah, H.O. (2012) Some Modifications of Adomian Decomposition Method Applied to Nonlinear System of Fredholm Integral Equations of the Second Kind. International Journal of Contemporary Mathematical Sciences, 7, 929-942.

[9] Bakodah, H.O. (2012) A New Modification of the Laplace Adomian Decomposition Method for System of Integral Equations. Journal of American Science, $\mathbf{8}$. 
[10] Bakodah, H.O. (2012) A Comparison Study between a Chebyshev Collocation Method and the Adomian Decomposition Method for Solving Linear System of Fredholm Integral Equations of the Second Kind. Journal of King Saud University: Science, 24, 49-59. https://doi.org/10.4197/Sci.24-1.4

[11] Khan, R.H. and Bakodah, H.O. (2013) Adomian Decomposition Method and Its Modification for Nonlinear Abel's Integral Equation. International Journal of Mathematical Analysis, 7, 2349-2358. https://doi.org/10.12988/ijma.2013.37179

[12] Bakodah, H.O., Al-Mazmumy, M. and Almuhalbedi, S.O. (2017) An Efficient Modification of the Adomian Decomposition Method for Solving Integro-Differential Equations. Mathematical Sciences Letters, 6, 15-21. https://doi.org/10.18576/msl/060103

[13] Khuri, S.A. (2001) A Laplace Decomposition Algorithm Applied to a Class of Nonlinear Differential Equations. Journal of Applied Mathematics, 1, 141-155. https://doi.org/10.1155/S1110757X01000183

[14] Khuri, S.A. (2004) A New Approach to Bratus Problem. Applied Mathematics and Computation, 147, 131-136. https://doi.org/10.1016/S0096-3003(02)00656-2

[15] Kiymaz, O. (2009) An Algorithm for Solving Initial Value Problems Using Laplace Adomian Decomposition Method. Applied Mathematical Sciences, 3, 1453-1459.

[16] Al-Mazmumy, M. and Al-Malki, H. (2015) Some Modification of Adomian Decomposition Method for Nonlinear Partial Differential Equations. International Journal of Research in Agricultural Sciences, 23, 164-173. https://doi.org/10.12988/nade.2015.41226

[17] Wazwaz, A. (2010) The Combined Laplace Transform-Adomian Decomposition Method for Handling Nonlinear Volterra Integro-Differential Equations. Applied Mathematics and Computation, 216, 1304-1309. https://doi.org/10.1016/j.amc.2010.02.023

[18] Bakodah, H.O. (2013) Adomian Decomposition Method and Its Modification for Nonlinear Abel's Integral Equation. International Journal of Mathematical Analysis, 7, 2349-2358. https://doi.org/10.12988/ijma.2013.37179

[19] Hussain, M. and Khan, M. (2010) Modified Laplace Decomposition Method. Applied Mathematical Sciences, 4, 1769-1783.

[20] Khan, Y. and Faraz, N. (2011) Application of Modified Laplace Decomposition Method for Solving Boundary Layer Equation. Journal of King Saud University (Science), 23, 115-119. https://doi.org/10.1016/j.jksus.2010.06.018

[21] Mahmoudi, M. and Jafari, H. (2014) Modified Laplace Decomposition Method for Singular IVPS in the Second-Order Ordinary Differential Equations. Caspian Journal of Mathematical Sciences, 3, 105-113.

[22] Manafianheris, J. (2012) Solving the Integro-Differential Equations Using the Modified Laplace Adomian Decomposition Method. Journal of Mathematica Extension, 6, 41-55.

[23] Behiry, S.H., Abd-Elmonem, R.A. and Gomaa, A.M. (2010) Discrete Adomian Decomposition Solution of Nonlinear Fredholm Integral Equation. Ain Shams Engineering Journal, 1, 97-101. https://doi.org/10.1016/j.asej.2010.09.009

[24] Bakodah, H.O. and Darwish, M.A. (2012) On Discrete Adomian Decomposition Method with Chebyshev Abscissa for Nonlinear Integral Equations of Hammerstein Type. Advances in Pure Mathematics, 2, 310-313. https://doi.org/10.4236/apm.2012.25042

[25] Bakodah, H.O. and Darwish, M.A. (2013) Solving Hammerstein Type Integral Equ- 
ation by New Discrete Adomian Decomposition Methods. Mathematical Problems in Engineering, 2013, Article ID: 760515. https://doi.org/10.1155/2013/760515

[26] Bakodah, H.O. and Darwish, M.A. (2014) Numerical Solutions of Quadratic Integral Equations. Life Science Journal, 11, 73-77. 